\title{
On identities in the products of group varieties
}

\author{
N. S. Boatman, A. Yu. Olshanskii *
}

July 16, 2018

\begin{abstract}
Let $\mathcal{B}_{n}$ be the variety of groups satisfying the law $x^{n}=1$. It is proved that for every sufficiently large prime $p$, say $p>10^{10}$, the product $\mathcal{B}_{p} \mathcal{B}_{p}$ cannot be defined by a finite set of identities. This solves the problem formulated by C.K. Gupta and A.N. Krasilnikov in 2003. We also find the axiomatic and the basis ranks of the variety $\mathcal{B}_{p} \mathcal{B}_{p}$. For this goal, we improve the estimate for the basis rank of the product of group varieties obtained by $\mathrm{G}$. Baumslag, B.H. Neumann, H. Neumann and P.M. Neumann long ago.
\end{abstract}

Key words : group identity, variety of groups, product of varieties, Burnside variety, $p$ group

AMS Mathematical Subject Classification: 20E10, 20E22, 20F05, 20F22, 20D15

\section{Introduction}

A variety of groups $\mathcal{V}$ is a class of groups given by a set of identical relations. For example, the variety of abelian groups $\mathcal{A}$ is given by the identity $[x, y]=1$, and the Burnside variety $\mathcal{B}_{n}$ contains all the groups with the identity $x^{n}=1$. In general, a group identity looks like $v\left(x_{1}, \ldots, x_{n}\right)=1$, where $v\left(x_{1}, \ldots, x_{n}\right)$ is a word, i.e., an element of a free group with a basis $x_{1}, \ldots, x_{n}, \ldots$

For an arbitrary group $G$, the verbal subgroup $V(G)$ is generated by all the values $v\left(g_{1}, \ldots, g_{n}\right)$ $\left(g_{1}, \ldots, g_{n} \in G\right)$ of the left-hand sides of the identities $v\left(x_{1}, \ldots, x_{n}\right)=1$ of the variety $\mathcal{V}$, and so $V(G)$ is the minimal normal subgroup $N$ of $G$ such that $G / N \in \mathcal{V}$. For example, $V(G)$ is the derived subgroup of $G$ if $\mathcal{V}=\mathcal{A}$ and $V(G)=G^{n}$ is generated by all the $n$-th powers of the elements from $G$ if $\mathcal{V}=\mathcal{B}_{n}$.

Given an arbitrary cardinal $m$, every variety $\mathcal{V}$ has a $\mathcal{V}$-free group $F_{m}(\mathcal{V})$ of free rank $m$ that is isomorphic to the factor group $F_{m} / V\left(F_{m}\right)$, where $F_{m}$ is the absolutely free group of rank $m$.

There are many varieties which are finitely based, that is, can be defined by finite sets of identities. For example, all nilpotent varieties $[\mathrm{L}$, all metabelian and nilpotent-by-abelian varieties $[\mathrm{C}, \mathrm{Kr}$, and every variety generated by a single finite group [OP] can be defined by a finite set of identities. We refer the reader to the book $[\mathrm{HN}]$ and also to the surveys $[\mathrm{BO}]$ and GK]. In the paper [N] (and also in his Ph.D. thesis in 1935), B.Neumann asked whether every group variety was finitely based. In 1969, the negative answers were, in turn, given in the papers [O70], [A] and [VL based on different methods.

A simplest example of an infinite system of group identities which is not equivalent to a finite one was presented in $[\mathrm{B}]$ and $[\mathrm{K} 73]$ :

$$
x_{1}^{8}=1,\left(x_{1}^{2} x_{2}^{2}\right)^{4}=1, \ldots,\left(x_{1}^{2} x_{2}^{2} \ldots x_{m}^{2}\right)^{4}=1, \ldots
$$

${ }^{*}$ The author was supported in part by the NSF grant DMS 1161294 
Note that a group $G$ satisfies the system of identities (1) if and only if it has a normal subgroup $N$ satisfying the identity $x^{4}=1$ such that the quotient $G / N$ satisfies the identity $x^{2}=1$. In other words, the set (1) defines the product $\mathcal{B}_{4} \mathcal{B}_{2}$ of two Burnside varieties $\mathcal{B}_{4}$ and $\mathcal{B}_{2}$. In general, the product $\mathcal{U V}$ of group varieties $\mathcal{U}$ and $\mathcal{V}$ consists of all groups $G$ having a normal subgroup $N \in \mathcal{U}$ such that $G / N \in \mathcal{V}$.

On one hand, an easy exercise shows that if positive integers $m$ and $n$ are relatively prime, then all the identities of the product $\mathcal{B}_{m} \mathcal{B}_{n}$ follow from the single identity $\left(x_{1}^{n} x_{2}^{n}\right)^{m}=1$. On the other hand, the following generalization of the example (11) is obtained in [K74]: For any prime $p$, the product $\mathcal{B}_{n} \mathcal{C}$ is not finitely based if $n$ is divisible by $p^{2}$ and the variety $\mathcal{C}$ contains a cyclic group of order $p$, where $\mathcal{C}$ is not the variety of all groups. The hypothesis $p^{2} \mid n$ was used in an essential way in [K74]; in particular, the case $\mathcal{B}_{p} \mathcal{B}_{p}$ remained unknown. The authors of the survey [GK] noticed that the variety $\mathcal{B}_{p} \mathcal{B}_{p}$ is finitely based for $p=2,3$, and they explicitly formulated the question (Problem 2 in $[\mathrm{GK}]$ ): "Is it true that for a large prime $p$ the variety defined by the identities

$$
x_{1}^{p^{2}}=1,\left(x_{1}^{p} x_{2}^{p}\right)^{p}=1, \ldots,\left(x_{1}^{p} x_{2}^{p} \ldots x_{m}^{p}\right)^{p}=1, \ldots
$$

is not finitely based ?"

Here we answer this question.

Theorem 1.1. For every sufficiently large prime $p$, the variety $\mathcal{B}_{p} \mathcal{B}_{p}$ is not finitely based, i.e. the system (2) is not equivalent to a finite system of identities.

This theorem can be formulated in a stronger form. Recall that a group variety $\mathcal{V}$ has infinite axiomatic rank if it cannot be given by any set of identities (finite or infinite) depending on a finite set of variables. A variety $\mathcal{V}$ has basis rank $d$ if it is generated by its $d$-generated groups, but is not generated by $(d-1)$-generated groups, i.e. the minimal variety containing all $(d-1)$-generated groups from $\mathcal{V}$ is strictly less than $\mathcal{V}$.

Theorem 1.2. For every sufficiently large prime $p$,

(1) the variety $\mathcal{B}_{p} \mathcal{B}_{p}$ has infinite axiomatic rank;

(2) its basis rank equal to 2.

The proof of statement (2) is based on Theorem 5.1 giving a new estimate of the basis ranks of the products $\mathcal{U} \mathcal{V}$ for various $\mathcal{U}$ and $\mathcal{V}$.

Remark 1.3. The methods of $\left[\mathrm{B}, \underline{\mathrm{K} 73}, \mathrm{~K} 74\right.$ ] work for the varieties $\mathcal{B}_{p^{2}} \mathcal{B}_{p}$ (and many other products of varieties) but cannot be applied to $\mathcal{B}_{p} \mathcal{B}_{p}$ for the following reason. The authors of $[\mathrm{B}$, K73, K74] check identities in solvable groups of bounded length and prove, in fact, that even the intersections $\mathcal{B}_{p^{2}} \mathcal{B}_{p} \cap \mathcal{S}$ have no finite bases of identities, where $\mathcal{S}$ is a finitely based solvable variety. But the variety $\mathcal{B}_{p} \mathcal{B}_{p} \cap \mathcal{S}=\left(\mathcal{B}_{p} \cap \mathcal{S}\right) \mathcal{B}_{p} \cap \mathcal{S}$ is finitely based because by G. Higman's theorem (see $[\mathrm{HN}], 34.23$ ), the product of the nilpotent variety $\mathcal{B}_{p} \cap \mathcal{S}$ and the finitely based variety $\mathcal{B}_{p}$ must be finitely based.

Our statement on the basis rank also contrasts with the locally finite case. If nontrivial varieties $\mathcal{U}$ and $\mathcal{V}$ consist of locally finite groups, then by Shmelkin's theorem $[\mathrm{S}]$, their product $\mathcal{U V}$ has finite basis rank if and only if all groups from $\mathcal{U}$ are nilpotent, all groups from $\mathcal{V}$ are abelian, and the exponents of $\mathcal{U}$ and $\mathcal{V}$ are relatively prime. 


\section{Auxiliary finite $p$-groups}

For an odd prime $p$ and positive integers $\ell, m$, we will consider the sets $U T(\ell, p, m)$ of $\ell \times \ell$ upper unitriangular matrices $A=\left(a_{i j}\right)$, where for $j-i=t>0$, the entry $a_{i j}$ is a $t$-linear function $V_{i} \times \cdots \times V_{j-1} \rightarrow \mathbb{F}_{p}$ to the finite Galois field $\mathbb{F}_{p}$, where $V_{1}, \ldots, V_{\ell-1}$ are $m$-dimensional vector spaces over $\mathbb{F}_{p}$ (and $a_{i i}=1$ ).

Assume that $A=\left(a_{i j}\right), B=\left(b_{i j}\right) \in U T(\ell, p, m)$. Then for $i \leq j \leq k$, the component-wise product of the mappings $a_{i j}$ and $b_{j k}$ is a $(k-i)$-linear mapping $V_{i} \times \ldots V_{j-1} \times V_{j} \times \cdots \times V_{k-1} \rightarrow$ $\mathbb{F}_{p}$. Therefore the matrix multiplication $A B=C$, where $c_{i k}=\sum_{j=1}^{\ell} a_{i j} b_{j k}$ is well defined in $U T(\ell, p, m)$. Moreover, it is associative for the ordinary reason, and every matrix $A=I+B$ is invertible in $U T(\ell, p, m):(I+B)^{-1}=I-B+\ldots \pm B^{\ell-1}$ since $B$ is a nil-triangle matrix. Hence the set $U T(\ell, p, m)$ is a finite group under the matrix multiplication. Moreover, it is a $p$-group since the number of possible entries $a_{i j}$ is a power of $p$ for every pair $(i, j)(1 \leq i<j \leq \ell)$. Similarly, all upper triangle matrices of this form (but the diagonal entries are any elements from $\mathbb{F}_{p}$ now) form an associative algebra over $\mathbb{F}_{p}$.

We will use the following properties of the groups $U T(p+1, p, m)$ (i.e. $\ell=p+1$ from now on) and of its subgroup $U T(p+1, p, m)^{p}$ generated by all the $p$ th powers of elements.

Lemma 2.1. (1) Let $A \in U T(p+1, p, m)$ and $C=A^{p}$. Then $c_{1, p+1}=a_{12} a_{23} \ldots a_{p, p+1}$ and other entries of the matrix $C$ coincide with the corresponding entries of the identity matrix $I$.

(2) $U T(p+1, p, m)^{p}$ is a nontrivial central subgroup of exponent $p$.

(3) If $p \geq 3$ and $m \geq 4$, then $U T(p+1, p, m)^{p}$ has a nontrivial cyclic subgroup containing no nontrivial element which can be presented in $U T(p+1, p, m)$ as a product of $m$ pth powers.

Proof. (1) We have $A=I+B$, where $B$ is a nil-triangular matrix. Hence $A^{p}=\sum_{i=0}^{p}\left(\begin{array}{c}p \\ i\end{array}\right) B^{i}=$ $I+B^{p}$ since $\left(\begin{array}{c}p \\ i\end{array}\right)$ is divisible by $p$ for $0<i<p$. Thus $c_{1, p+1}=b_{12} b_{23} \ldots b_{p, p+1}=a_{12} a_{23} \ldots a_{p, p+1}$ and other entries of $B^{p}$ are 0 , which proves the statement.

(2) It follows from the claim (1) that there is a non-identity matrix $C$ in $U T(p+1, p, m)^{p}$. It is straightforward that every matrix $C$ which is equal to $I$, except possibly in the entry $c_{1, p+1}$, belongs to the center of $U T(p+1, p, m)$. Also we have $C^{p}=I$ for such a matrix since $C$ has zeros just above the diagonal. This proves the statement (2).

(3) On one hand, each of the vector spaces $V_{i}$ has $p^{m}$ elements, and so there are $p^{m}$ linear functions on $V_{i}$ with values in $\mathbb{F}_{p}$. Hence the number of different products $a_{12} \ldots a_{p, p+1}$ does not exceed $\left(p^{m}\right)^{p}=p^{p m}$. It follows from the claim (1) that the number of different products of the form $A_{1}^{p} \ldots A_{m}^{p}$ in $U T(p+1, p, m)$ does not exceed $\left(p^{p m}\right)^{m}=p^{p m^{2}}$.

On the other hand, the vector space of $p$-linear mappings $V_{1} \times \cdots \times V_{p} \rightarrow \mathbb{F}_{p}$ has dimension $m^{p}$ over $\mathbb{F}_{p}$. This space is a linear envelope of $m^{p}$ linearly independent products of $p$ basic 1linear mappings from $V_{i}$ to $\mathbb{F}_{p}(i=1, \ldots, p)$. Therefore the claim (1) implies that the subgroup $U T(p+1, p, m)^{p}$ has dimension $m^{p}$ over $\mathbb{F}_{p}$, and so its order is $p^{m^{p}}$.

It follows that the subgroup $U T(p+1, p, m)^{p}$ has $\left(p^{m^{p}}-1\right) /(p-1)$ subgroups of order $p$. Since the pairwise intersections of these cyclic subgroups are trivial, one of the subgroups contains no nontrivial products of the form $A_{1}^{p} \ldots A_{m}^{p}$ if $\left(p^{m^{p}}-1\right) /(p-1)>p^{p m^{2}}$. The last inequality follows from the inequalities $p \geq 3$ and $m \geq 4$, and the proof is complete.

\section{Construction of infinite torsion groups}

Our proof of Theorem 1.1 makes use the approach from [089]. We recall a few definitions here. 
Given a finite or infinite group alphabet $\mathcal{A}^{ \pm 1}=\left\{a_{1}^{ \pm 1}, a_{2}^{ \pm 1}, \ldots\right\}$, we we write $U \equiv V$ to express letter-by-letter equality of two words $U$ and $V$ over $\mathcal{A}^{ \pm 1}$. If $\mathcal{A}$ is a generating set of a group $G$, we write $U=V$ whenever two words $U$ and $V$ over $\mathcal{A}^{ \pm 1}$ represent the same element of $G$; we identify the words over $\mathcal{A}^{ \pm 1}$ and the elements of $G$ represented by them.

We shall assume that the series of groups $G(0), G(1), \ldots$ is defined according to the following scheme. By definition, $G(0)=F(\mathcal{A})$ is the free group with basis $\mathcal{A}$. We also set $\mathcal{R}_{0}=\mathcal{X}_{0}=\emptyset$.

For $i \geq 1$, we assume by induction that the set of defining relators $\mathcal{R}_{i-1}$ of the group $G(i-1)=\left\langle\mathcal{A} \mid \mathcal{R}_{i-1}\right\rangle$ is already defined, as well as the sets $\mathcal{X}_{j}$ of periods of ranks $j<i$.

For $i \geq 1$, a word $X$ in the alphabet $\mathcal{A}^{ \pm 1}$ is called simple in rank $i-1$, if it is not conjugate to a power of a shorter word or to a power of a period of rank $\leq i-1$ in the group $G(i-1)$. We denote by $\mathcal{X}_{i}$ a maximal subset of words satisfying the following conditions.

1) $\mathcal{X}_{i}$ consists of words of length $i$ which are simple in rank $i-1$.

2) If $A, B \in \mathcal{X}_{i}$ and $A \not \equiv B$, then $A$ is not conjugate to $B$ or $B^{-1}$ in the group $G(i-1)$.

Each word from $\mathcal{X}_{i}$ is called a period of rank $i$. For every period $A \in \mathcal{X}_{i}$ we fix an odd integer $n_{A}$ which should be large enough, i.e. $n_{A} \geq n_{0}$ for a large constant $n_{0}$.

Let $\mathcal{S}_{i}=\left\{A^{n_{A}} \mid A \in \mathcal{X}_{i}\right\}$. Then the set $\mathcal{R}_{i}$ is defined by $\mathcal{R}_{i}=\mathcal{R}_{i-1} \cup \mathcal{S}_{i}$, and so $G(i)=\langle\mathcal{A}|$ $\left.\mathcal{R}_{i}\right\rangle$. By definition, the group $G(\infty)$ is the inductive limit for the epimorphisms $G(0) \rightarrow G(1) \rightarrow$ $\ldots$, i.e., $G(\infty)=\left\langle\mathcal{A} \mid \cup_{i=0}^{\infty} \mathcal{R}_{i}\right\rangle$.

The group $G(\infty)$ depends on the choice of the exponents $n_{A}$, but there are some general properties for such constructions.

Lemma 3.1. (1) ([089], Theorem 26.4.3) Every element of $G(\infty)$ is conjugate to a power of a period of some rank $i \geq 1$.

(2) ([089], Theorem 26.4.1) Every period $A$ has order $n_{A}$ in $G(\infty)$.

\section{Main construction}

To combine the constructions from Sections 2 and 3 we fix a prime $p \geq n_{0}$, fix an integer $m \geq 4$ and consider the finite $p$-group $U=U T(p+1, p, m)$. By Lemma 2.1 (3), the subgroup $U^{p}$ has an element $g$ such that no nontrivial element of $\langle g\rangle$ is a product of $m p$ th powers in $U$. Then we take a free group $F$ admitting an epimorphism $f: F \rightarrow U$. Let $H=F^{p}$ be the subgroup generated by all $p$ th powers in $F$. Its image $f(H)=f\left(F^{p}\right)=f(F)^{p}=U^{p}$ is a central subgroup of $U$ by Lemma 2.1 (2).

Being a free group itself, the subgroup $H$ has a free basis $\mathcal{A}$. So one can construct the groups $G(0), G(1), \ldots$ as in Section 3, starting with $G(0)=H$. We will use the mapping $f$ to choose the exponents $n_{A}$ of the periods as follows. If $A$ is a period of some rank $i \geq 1$ and $f(A) \in U^{p} \backslash\langle g\rangle$ or $f(A)=1$, then $n_{A}=p$; otherwise $n_{A}=p^{2}$.

Let $N=N(m)$ be the kernel of the canonical mapping $H \rightarrow G(\infty)=\langle\mathcal{A} \mid \mathcal{R}\rangle$.

Lemma 4.1. If two words $V$ and $W$ over $\mathcal{A}^{ \pm 1}$ are equal in $G(\infty)$, then $f(V)=f(W)$.

Proof. By the definition of the exponents $n_{A}$ and Lemma 2.1 (2), every relator $A^{n_{A}}$ belongs to $\operatorname{Ker} f$. Hence $N \leq \operatorname{Ker} f$, and the statement follows.

Lemma 4.2. Let $B$ be a word in the alphabet $\mathcal{A}^{ \pm 1}$ and $B \neq 1$ in $G(\infty)$.

(1) If $f(B) \in U^{p} \backslash\langle g\rangle$ or $f(B)=1$, then $B$ has order $p$ in $G(\infty)$.

(2) If $f(B) \in\langle g\rangle \backslash\{1\}$, then the order of $B$ in $G(\infty)$ is $p^{2}$. 
Proof. By Lemma 3.1 (1), $B$ is conjugate to a power of a period $A$ in $G(\infty)$; say $B=Z A^{k} Z^{-1}$, and so the order of $B$ is equal to the order of $A^{k}$. The conditions $f(B) \in U^{p} \backslash\langle g\rangle$ and $f\left(Z A^{k} Z^{-1}\right) \in U^{p} \backslash\langle g\rangle$ are equivalent by Lemma 4.1. So are the conditions $f(B)=1$ and $f\left(Z A^{k} Z^{-1}\right)=1$.

The condition $f\left(Z A^{k} Z^{-1}\right) \in U^{p} \backslash\langle g\rangle$ is equivalent to $f\left(A^{k}\right) \in U^{p} \backslash\langle g\rangle$ by Lemma 4.1. Similarly, the condition $f(B) \in\langle g\rangle \backslash\{1\}$ is equivalent to $f\left(A^{k}\right) \in\langle g\rangle \backslash\{1\}$. Thus one may just assume that $B \equiv A^{k}$.

(1) Assume that $f\left(A^{k}\right) \in U^{p} \backslash\langle g\rangle$ or $f(B)=1$. If $(p, k)=1$, then both $A^{k}$ and $A$ generate the $p$-subgroup $\langle A\rangle$. The same is true for the pair $f\left(A^{k}\right)$ and $f(A)$ since $U$ is a $p$-group. Thus the order of $A^{k}$ is equal to the order of $A$ in $G(\infty)$, which is $p$ by Lemma 3.1 (2). If $p$ divides $k$, then the order of $A^{k}$ is $p$ since $A^{p^{2}}=1$ for any period $A$ and $A^{k}$ is nontrivial in $G(\infty)$.

(2) Now suppose $f\left(A^{k}\right) \in\langle g\rangle \backslash\{1\}$. Since no element of $\langle g\rangle \backslash\{1\}$ is a $p$-th power (moreover, it cannot be a product of $m p$ th powers in $U)$, we have $(p, k)=1$. Thus, as above, one can replace $A^{k}$ by $A$ in the proof, and the order of $A$ is $n_{A}=p^{2}$ since $f(A) \in\langle g\rangle \backslash\{1\}$. The lemma is proved.

Lemma 4.3. The subgroup $N=N(m)$ is normal in the group $F$.

Proof. By definition, $N$ is the normal closure in $H$ of the powers $A^{n_{A}}$ of periods, where $n_{A}=p$ if $f(A) \in U^{p} \backslash\langle g\rangle$ or $f(A)=1$, and $n_{A}=p^{2}$ otherwise. By the statement (1), $N$ is the normal closure in $H$ of a bigger set $\mathcal{Q}$; namely, $\mathcal{Q}$ consists of $p$-th powers of all the words $B$, where $f(B) \in U^{p} \backslash\langle g\rangle$ or $f(B)=1$, and of $p^{2}$-th powers of all other words. Since $\langle g\rangle$ is a normal subgroup of $U$ by Lemma 2.1 (2), the set $\mathcal{Q}$ is invariant under conjugations in $F$. Therefore the subgroup $N$ generated by $\mathcal{Q}$ is normal in $F$.

Denote by $L(m)$ the quotient $F / N(m)$. Now we are prepared to prove the main lemma.

Lemma 4.4. For given $m \geq 4$ the group $L(m)$ does not belong to the variety $\mathcal{B}_{p} \mathcal{B}_{p}$ but it satisfies the identical relation $\left(x_{1}^{p} \ldots x_{m}^{p}\right)^{p}=1$.

Proof. The group $H=F^{p}$ has an element $B$ such that $f(B) \in\langle g\rangle \backslash\{1\}$. Its order in $H / N \leq$ $F / N=L(m)$ is $p^{2}$ by Lemma $4.2(2)$. Therefore the verbal subgroup $(F / N)^{p}$ of $F / N$ does not belong to the variety $\mathcal{B}_{p}$, and so $F / N \notin \mathcal{B}_{p} \mathcal{B}_{p}$.

If $h=g_{1}^{p} \ldots g_{m}^{p}$ for some $g_{1}, \ldots, g_{m} \in F$, then $f(h)=f\left(g_{1}\right)^{p} \ldots f\left(g_{m}\right)^{p} \in U^{p}$. So either $f(h)=1$ or $f(h) \notin\langle g\rangle$ by the definition of the element $g$. Hence $h^{p}=1$ in $H / N$ by Lemma 4.2 (1). Since $\left(g_{1}^{p} \ldots g_{m}^{p}\right)^{p}=1$ in $F / N$ for every $m$-tuple $\left(g_{1}, \ldots, g_{m}\right)$, the required identity is obtained.

Proof of Theorem 1.1. If the system (2) is equivalent to a finite system of identities, then it is equivalent to its own finite subsystem

$$
x_{1}^{p^{2}}=1,\left(x_{1}^{p} x_{2}^{p}\right)^{p}=1, \ldots,\left(x_{1}^{p} x_{2}^{p} \ldots x_{m}^{p}\right)^{p}=1
$$

for some $m$, and one may assume that $m \geq 4$. Note that the last identity implies all the preceding ones. By Lemma 4.4, there is a group $L(m)$ satisfying the system (3) but not satisfying the system (2). Thus, the theorem is proved by contradiction.

Proof of part (1) in Theorem 1.2. It suffices for every $k \geq 1$, to find a group $M(k)$ which does not belong to the product $\mathcal{B}_{p} \mathcal{B}_{p}$ but every $k$-generated subgroup of $M(k)$ lies in $\mathcal{B}_{p} \mathcal{B}_{p}$.

Note that for given $k$, all $k$-generated subgroups $K$ of the groups $U T(p+1, p, m)$ ( $m=$ $1,2, \ldots)$ have bounded orders since their nilpotency classes, exponents and the numbers of 
generators are bounded (by $p, p^{2}$ and $k$, respectively). Therefore there exists $m=m(k)$ such that every element of $K^{p}$ is a product of at most $m p$-th powers in every such a subgroup $K$.

Let now $M(k)=G(\infty)$ be the group whose definition in Section 4 depends on the group $U=U(p+1, p, m)$ and the choice of $g \in U^{p}$, where nontrivial elements of $\langle g\rangle$ are not products of $m p$-th powers in $U$. It follows that $K^{p} \cap\langle g\rangle=\{1\}$ for every $k$-generated subgroup $K$ of $U$.

Assume now that $L$ is a $k$-generated subgroup of $M(k)$. Since $f(L)$ is a $k$-generated subgroup of $U$, we have $f(L)^{p} \cap\langle g\rangle=\{1\}$. Therefore by Lemma 4.2, we have $z^{p}=1$ for every element $z$ of the subgroup $L^{p}$, that is, the subgroup $L$ satisfies the system of identities (2). Since by Lemma 4.4, the group $M(k)$ itself does not belong to the variety $\mathcal{B}_{p} \mathcal{B}_{p}$, the proof of the statement (1) is complete.

\section{Products of varieties and their basis rank}

For the second part of Theorem 1.2, we will use the concept introduced in BNNN]. A group $G$ is said to be discriminated by a group $D$ if for every finite subset $S \subset G$, there is a homomorphism $G \rightarrow D$ injective on $S$. If a free group of infinite countable rank $F_{\infty}(\mathcal{V})$ of a variety $\mathcal{V}$ is discriminated by a group $D \in \mathcal{V}$, then $\operatorname{var} D=\mathcal{V}$, i.e. the variety $\mathcal{V}$ is generated by the group $D$. Clearly a group $C$ discriminates a group $G$ if a subgroup $D \leq C$ discriminates $G$. If a factor group $B$ of $D$ discriminates the group $F_{\infty}(\mathcal{V})$ and $D \in \mathcal{V}$, then $D$ discriminates the group $F_{\infty}(\mathcal{V})$ too since every homomorphism $F_{\infty}(\mathcal{V}) \rightarrow B$ lifts to a homomorphism $F_{\infty}(\mathcal{V}) \rightarrow D$ by the universal property of $\mathcal{V}$-free groups.

Theorem 5.1. Let a group $G$ be $m$-generated, $m \geq 2$, such that $G$ belongs to a variety $\mathcal{V}$. If $G$ contains a finitely generated subgroup $D$ of infinite index which discriminates the $\mathcal{V}$-free group $F_{\infty}(\mathcal{V})$, then for an arbitrary variety of groups $\mathcal{U}$, the $\mathcal{U V}$-free group $F_{\infty}(\mathcal{U V})$ is discriminated by an m-generated group from $\mathcal{U V}$. Therefore the basis rank of the product $\mathcal{U} \mathcal{V}$ does not exceed $m$.

Proof. One may assume that $\mathcal{V} \neq \mathcal{O}$, where $\mathcal{O}$ is the variety of all groups, because $\mathcal{U O}=\mathcal{O}$ and the (absolutely) free group $F_{m}$ of rank $m$ discriminates the group $F_{\infty}=F_{\infty}(\mathcal{O})$ since $F_{m}$ contains an isomorphic copy of $F_{\infty}$.

Let $N$ be a normal subgroup of $F_{m}$ such that $F_{m} / N \cong G$. We have $N \neq\{1\}$ since $G \in \mathcal{V} \neq \mathcal{O}$. The group $F_{m} / U(N)$, where $U(N)$ is the verbal subgroup of $N$ corresponding to the variety $\mathcal{U}$, obviously belongs to the variety $\mathcal{U} \mathcal{V}$. It follows from the assumptions that there is a subgroup $K \leq F_{m}$ such that $N \leq K, K / N \cong D$ and the index $\left[F_{m}: K\right]$ is infinite.

The group $K$ is free, being a subgroup of $F_{m}$, and $K$ is not finitely generated since it has infinite index in $F_{m}$ and contains a nontrivial normal subgroup $N$ of $F_{m}$ (see [LS], Proposition I.3.11). Thus we have an infinite free basis $y_{1}, y_{2}, \ldots$ in $K$.

Since $K / N$ is a finitely generated group, there is a finite subset $y_{1}, \ldots, y_{s}$ generating the subgroup $K$ modulo $N$. Therefore there are words $v_{s+1}=v_{s+1}\left(y_{1}, \ldots, y_{s}\right), v_{s+2}=v_{s+2}\left(y_{1}, \ldots, y_{s}\right), \ldots$ such that the products $z_{1}=y_{s+1} v_{s+1}, z_{2}=y_{s+2} v_{s+2}, \ldots$ belong to $N$. The set $y_{1}, \ldots, y_{s}, z_{1}, \ldots$ is also a free basis of $K$, since it is obtained from a basis by Nielsen transformations.

Now we consider the auxiliary wreath product $W=F_{\infty}(\mathcal{U}) w r D$ which belongs to the product $\mathcal{U V}$ and discriminates the group $F_{\infty}(\mathcal{U V})$ (see [Ba] or [HN], Corollary 22.44). Recall that $W$ is generated by a copy $A$ of $F_{\infty}(\mathcal{U})$ and by $D, W$ is a semidirect product of the normal closure $M$ of $A$ in $W$ and the subgroup $D, M$ is isomorphic to a direct power of $A$, and so $M \in \mathcal{U}$.

Let $\varepsilon$ be the canonical homomorphism $K \rightarrow K / N=D$. To construct an epimorphism $\eta: K \rightarrow W$, we will modify $\varepsilon$ as following. The elements of the free basis $y_{1}, \ldots, y_{s}$ of $K$ are 
mapped by $\eta$ into $D$ according to $\varepsilon$ but the set $\left\{z_{1}, z_{2}, \ldots\right\}$ is mapped onto a generating set of $A$. The canonical projection $\alpha$ of $W$ onto the semidirect factor $D$ removes the difference between $\eta$ and $\varepsilon$, that is, $\alpha \eta(x)=\varepsilon(x)$ for every $x \in\left\{y_{1}, \ldots, y_{s}, z_{1}, \ldots\right\}$. It follows that $\eta(N) \leq \operatorname{ker} \alpha=M$.

Since $\eta(N) \leq M \in \mathcal{U}$, we have $\eta(U(N))=U(\eta(N))=\{1\}$. Hence the epimorphism $\eta$ factors through an epimorphism $K / U(N) \rightarrow W$. Since $W$ discriminates the group $F_{\infty}(\mathcal{U V})$, so do the group $K / U(N)$ and the $m$-generated group $F_{m} / U(N)$ containing $K / U(N)$. This completes the proof of Theorem 5.1 .

Corollary 5.2. For any variety $\mathcal{U}$ and sufficiently large odd integer $n$, the variety $\mathcal{U} \mathcal{B}_{n}$ has basis rank 2.

Proof. Recall that for large enough odd $n$ the group $F_{\infty}\left(\mathcal{B}_{n}\right)$ is isomorphic to a subgroup of $F_{2}\left(\mathcal{B}_{n}\right)$ (see [Sh or [089], Corollary 35.6). Hence $F_{\infty}\left(\mathcal{B}_{n}\right)$ is discriminated by the 2-generated group $F_{2}\left(\mathcal{B}_{n}\right)$, and the group $F_{2}\left(\mathcal{B}_{n}\right)$ contains its own isomorphic copy as a subgroup of infinite index. Therefore the basis rank of the product $\mathcal{U} \mathcal{B}_{n}$ is at most 2 by Theorem 5.1. Clearly, it is greater than 1 , since this product is not an abelian variety.

Proof of part (2) in Theorem 1.2. The statement follows from Corollary 5.2 with $\mathcal{U}=\mathcal{B}_{p}$ and $n=p . \square$.

Remark 5.3. One can replace "large enough odd" by "divisible by a large enough power of 2" in the formulation of Corollary 5.2 since under the latter assumption, the group $F_{\infty}\left(\mathcal{B}_{n}\right)$ also embeds into the group $F_{2}\left(\mathcal{B}_{n}\right)[\mathrm{IO}]$.

Corollary 5.4. If a variety $\mathcal{V}$ is nontrivial and the group $F_{\infty}(\mathcal{V})$ is discriminated by an $m$ generated group $D$ from $\mathcal{V}$, then for every variety $\mathcal{U}$, the group $F_{\infty}(\mathcal{U V})$ is discriminated by an $(m+1)$-generated group from $\mathcal{U} \mathcal{V}$. In particular, the basis rank of the product $\mathcal{U} \mathcal{V}$ does not exceed $m+1$.

Proof. The group $D$ is a homomorphic image of the $\mathcal{V}$-free group $F_{m}(\mathcal{V})$, and so $F_{m}(\mathcal{V})$ discriminates the group $F_{\infty}(\mathcal{V})$ as well. It is naturally embedded in $F_{m+1}(\mathcal{V})$ as a subgroup of infinite index. Indeed if we had $\left[F_{m+1}(\mathcal{V}): F_{m}(\mathcal{V})\right]<\infty$ and $\left(a_{1}, \ldots a_{m+1}\right)$ was a $\mathcal{V}$-free basis of $F_{m+1}(\mathcal{V})$, then the endomorphism $a_{1} \mapsto 1, a_{2} \mapsto a_{1}, \ldots a_{m+1} \mapsto a_{m}$ would show that $\left[F_{m}(\mathcal{V}): F_{m-1}(\mathcal{V})\right]<\infty$ as well. By induction, it would follow that $\left|F_{m+1}(\mathcal{V})\right|<\infty$, contrary to the fact that a nontrivial discriminating group cannot be finite $([\mathrm{HN}], 17.32)$. Hence the corollary follows from Theorem 5.1 applied to $F_{m+1}(\mathcal{V})$.

Remark 5.5. On one hand, for any two group varieties $\mathcal{U}$ and $\mathcal{V}$ with $\mathcal{U} \neq \mathcal{O}$, where $\mathcal{O}$ is the variety of all groups, the basis rank of $\mathcal{V}$ does not exceed the basis rank of the product $\mathcal{U V}([\mathrm{HN}]$, 25.12). On the other hand, the free abelian group of any rank is discriminated by an infinite cyclic group $([\mathrm{HN}], 17.6)$. Hence $m=1$ if $\mathcal{V}$ is the variety of all abelian groups. But for any nontrivial variety $\mathcal{U}$, the product $\mathcal{U} \mathcal{V}$ is not abelian, and so its basis rank is at least 2 . Therefore the statement of Corollary 5.4 fails when replacing $m+1$ by $m$ in its formulation.

Note that the weaker formulation with $2 m+1$ instead of $m+1$ can be found in [BNNN] and $[\mathrm{HN}]$, Corollary 25.22. H.Neumann mentioned in $[\mathrm{HN}]$ that she needed additional assumptions to get $(m+1)$-generated group discriminating the group $F_{\infty}(\mathcal{U V})$. Since Corollary 5.4 gives the estimate $m+1$ without any additional restrictions, Theorem 5.1 is of certain interest independently of the application to Theorem 1.2 . 


\section{References}

[A] S.I. Adyan, Infinite irreducible system of group identities, Izv. Akad. Nauk SSSR, Ser. Math. 34 (1970), no. 4, 376-384; English translation in Math. USSR Izv.

[BO] Yu.A. Bahturin, A.Yu. Olshanskii, Identities, English translation in Encyclopaedia of Mathematical Sciences, Algebra II, v. 18, Springer-Heidelberg, 1991, 107-221.

[Ba] G. Baumslag, Wreath products and extensions, Math. Z., 81 (1963), 286-289.

[BNNN] G. Baumslag, B.H. Neumann, H. Neumann, P.M. Neumann, On the varieties generated by finitely generated group, Math. Z., 86 (1964), 93-122.

[B] R.M. Bryant, Some infinitely based varieties of groups, J. Austral. Math. Soc. 16 (1973), 29-32.

[C] D.E. Cohen, On the laws of metabelian variety, J. algebra, 5 (1967), 267-273.

[GK] C.K. Gupta, A.N. Krasilnikov, The finite basis question for varieties of groups - Some recent results, Illinois Journal of Mathematics, 47 (2003), no. 1-2, 273-283.

[IO] S.V. Ivanov, A. Yu. Olshanskii, On finite and locally finite subgroups of free Burnside groups of large even exponents, Journal of Algebra, 195(1997), 241-284.

[K73] Yu.G. Kleiman, The basis of a product of group varieties I, Izv. Akad. Nauk SSSR, Ser. Math. 37 (1973), 475-483; English translation in Math. USSR Izv.

[K74] Yu.G. Kleiman, On the basis of a product of group varieties II, Izv. Akad. Nauk SSSR, Ser. Math. 38 (1974), 95-97; English translation in Math. USSR Izv.

[Kr] A.N. Krasilnikov, On the finitness of the basis of identities of groups with nilpotent commutator subgroup, Izv. Akad. Nauk SSSR, Ser. Math. 54 (1990), 1181-1195; English translation in Math. USSR Izv.

[L] R.C. Lyndon, Two notes on nilpotent groups, Proc. AMS, 3 (1952), 579-583.

[LS] R.C. Lyndon and P.E. Schupp, Combinatorial Group Theory, Springer-Verlag, 2001.

[N] B. Neumann, Identical relations in groups I, Math. Ann., 114 (1937), 506-525.

[HN] H. Neumann, Varieties of groups, Springer, Berlin-Heidelberg-New York, 1967.

[OP] Sh. Oates, M.B. Powell, Identical relations in finite groups, J. Algebra, 1 (1964), no. 1, 11-39.

[O70] A.Yu. Olshanskii, On the problem of finite basis of identities in groups, Izv. Akad. Nauk SSSR, Ser. Math. 34 (1970), no. 2, 376-384; English translation in Math. USSR Izv.

[O89] A.Yu. Olshanskii, Geometry of defining relations in groups, Moscow, Nauka, 1989, 448 pp. (in Russian); English translation by Kluwer Academic Publishers, 1991.

[Sh] V.L. Shirvanyan, Embedding of the group $B(\infty, n)$ into the group $B(2, n)$, Izv. Akad. Nauk SSSR, Ser. Math. 40 (1976), no. 1, 190-208; English translation in Math. USSR Izv. 
[S] A.L. Shmelkin, Wreath products and varieties of groups, Izv. Akad. Nauk SSSR, Ser. Math. 29 (1965), 149-170; English translation in Math. USSR Izv.

[VL] M.R. Vaughan-Lee, Uncountably many varieties of groups, Bull. London Math. Soc. 2 (1970), 280-286.

Nicholas S. Boatman: Vanderbilt University, 2012. E-mail: nickboatman@yahoo.com

Alexander A. Olshanskii: Vanderbilt University, Nashville 37240, USA, and Moscow State University, Moscow 119991, Russia..E-mail: alexander.olshanskiy@vanderbilt.edu 